\title{
On certain subclasses of analytic functions involving a linear operator
}

\author{
by J. Patel and G. P. Mohapatra (Bhubaneswar)
}

\begin{abstract}
A certain general class $\mathcal{S}(a, c, A, B)$ of analytic functions involving a linear operator is introduced. The objective is to investigate various properties and characteristics of this class. Several applications of the results (obtained here) to a class of fractional calculus operators are also considered. The results contain some of the earlier work in univalent function theory.
\end{abstract}

1. Introduction. Let $\mathcal{A}$ denote the class of functions of the form

$$
f(z)=z+\sum_{n=2}^{\infty} a_{n} z^{n}
$$

that are analytic in the open unit disc $E=\{z:|z|<1\}$. Let $\mathcal{S}, \mathcal{S}^{*}(\alpha)$ and $\mathcal{K}(\alpha)(0 \leq \alpha<1)$ be the usual subclasses of functions in $\mathcal{A}$ that are univalent, starlike of order $\alpha$ and convex of order $\alpha$, respectively. We note that

$$
f(z) \in \mathcal{K}(\alpha) \Leftrightarrow z f^{\prime}(z) \in \mathcal{S}^{*}(\alpha) .
$$

For arbitrary fixed real numbers $A, B(-1 \leq B<A \leq 1)$, let $\mathcal{P}(A, B)$ denote the class of functions of the form

$$
\phi(z)=1+c_{1} z+c_{2} z^{2}+\ldots
$$

which are analytic in $E$ and satisfy the condition

$$
\phi(z) \prec \frac{1+A z}{1+B z} \quad(z \in E)
$$

where the symbol $\prec$ stands for subordination. The class $\mathcal{P}(A, B)$ was introduced and studied by Janowski [5].

2000 Mathematics Subject Classification: Primary 30C45.

Key words and phrases: differential subordination, hypergeometric functions, Hadamard product (or convolution). 
For a function $f \in \mathcal{A}$ given by (1.1), the generalized Bernardi integral operator $\mathcal{F}_{\delta}$ is

$$
\mathcal{F}_{\delta}(z)=\frac{\delta+1}{z^{\delta}} \int_{0}^{z} t^{\delta-1} f(t) d t=z+\sum_{n=2}^{\infty} \frac{\delta+1}{\delta+n} a_{n} z^{n} \quad(\delta>-1, z \in E) .
$$

It readily follows from (1.2) that

$$
f(z) \in \mathcal{A} \Leftrightarrow \mathcal{F}_{\delta}(z) \in \mathcal{A} .
$$

Several essentially equivalent definitions of fractional calculus have been given in the literature (cf., e.g., [12], [13], [14]). We state the following definitions due to Owa [10] which have been used rather frequently in the theory of analytic functions.

Definition 1. The fractional integral of order $\lambda$ is defined, for a function $f(z)$, by

$$
D_{z}^{-\lambda} f(z)=\frac{1}{\Gamma(\lambda)} \int_{0}^{z} \frac{f(\zeta)}{(\zeta-z)^{1-\lambda}} d \zeta \quad(\lambda>0)
$$

and the fractional derivative of order $\lambda$ is defined by

$$
D_{z}^{\lambda} f(z)=\frac{1}{\Gamma(1-\lambda)} \frac{d}{d z} \int_{0}^{z} \frac{f(\zeta)}{(\zeta-z)^{\lambda}} d \zeta \quad(0 \leq \lambda<1),
$$

where $f(z)$ is analytic in a simply connected region of the $z$-plane containing the origin, and the multiplicity of $(z-\zeta)^{\lambda-1}$ involved in (1.3) (and that of $z-\zeta$ involved in (1.4)) is removed by requiring $\log (z-\zeta)$ to be real when $z-\zeta>0$.

Definition 2. Under the hypotheses of Definition 1, the fractional derivative of order $n+\lambda$ is defined by

$$
D_{z}^{n+\lambda} f(z)=\frac{d^{n}}{d z^{n}}\left(D_{z}^{\lambda} f(z)\right) \quad\left(0 \leq \lambda<1, n \in \mathbb{N}_{0}=\{0,1, \ldots\}\right) .
$$

Let

$$
\phi(a, c ; z)=\sum_{n=0}^{\infty} \frac{(a)_{n}}{(c)_{n}} z^{n+1} \quad(c \neq 0,-1,-2, \ldots)
$$

where $(x)_{n}$ is the Pochhammer symbol defined by

$$
(x)_{n}=\frac{\Gamma(x+n)}{\Gamma(x)}= \begin{cases}x(x+1)(x+2) \ldots(x+n-1), & n \in \mathbb{N}_{0}, \\ 1, & n=0 .\end{cases}
$$

We note that $\phi(a, 1 ; z)=z /(1-z)^{a}$ and $\phi(2,1 ; z)$ is the well known Koebe function. 
Corresponding to the function $\phi(a, c ; z)$ and for an analytic function $f(z)$ given by (1.1), Carlson and Shaffer [4] defined a linear operator $\mathcal{L}(a, c)$ by

$$
\mathcal{L}(a, c) f(z)=\phi(a, c ; z) * f(z)=z+\sum_{n=2}^{\infty} \frac{(a)_{n-1}}{(c)_{n-1}} a_{n} z^{n}
$$

where the symbol $*$ stands for the Hadamard product (or convolution). We see that if $a=0,-1,-2, \ldots$, then $\mathcal{L}(a, c) f(z)$ is a polynomial. For $a \neq 0,-1,-2, \ldots$, an application of the root test shows that the infinite series for $\mathcal{L}(a, c) f(z)$ has the same radius of convergence as that of $f(z)$ because $\lim _{n \rightarrow \infty}\left|(a)_{n} /(c)_{n}\right|^{1 / n}=1$. Hence, $\mathcal{L}(a, c)$ maps $\mathcal{A}$ into itself. The Ruscheweyh derivatives [11] of $f(z)$ are $\mathcal{L}(n+1,1) f(z), n \in \mathbb{N}_{0}$.

We further observe that

$\mathcal{L}(a, a) f(z)=f(z), \quad \mathcal{L}(2,1) f(z)=z f^{\prime}(z), \quad \mathcal{L}(\delta+1, \delta+2) f(z)=F_{\delta}(z)$ and

$$
\mathcal{L}(2,2-\lambda) f(z)=\Gamma(2-\lambda) z^{\lambda} D_{z}^{\lambda} f(z)=J_{z}^{\lambda} f(z) \quad(0 \leq \lambda<1) .
$$

Making use of the operator $\mathcal{L}(a, c)$, we now introduce a subclass of $\mathcal{A}$ as follows:

Definition 3. A function $f \in \mathcal{A}$ is said to be in the class $\mathcal{S}(a, c, A, B)$ if it satisfies

$$
\frac{\mathcal{L}(a, c-1) f(z)}{\mathcal{L}(a, c) f(z)} \prec \frac{1+A z}{1+B z} \quad(z \in E)
$$

for some $a>0, c>1$, and $-1 \leq B<A \leq 1$. By the definition of subordination, it follows that

$$
\left|\frac{\mathcal{L}(a, c-1) f(z)-\mathcal{L}(a, c) f(z)}{A \mathcal{L}(a, c) f(z)-B \mathcal{L}(a, c-1) f(z)}\right|<1 \quad(z \in E) .
$$

For convenience, we put

$$
\mathcal{S}(2,2-\lambda, \beta(1-2 \alpha),-\beta)=\mathcal{S}(\lambda, \alpha, \beta) \quad(0 \leq \lambda<1,0 \leq \alpha<1,0<\beta \leq 1),
$$

the class consisting of functions in $\mathcal{A}$ satisfying the condition

$$
\left|\frac{J_{z}^{\lambda+1} f(z)-J_{z}^{\lambda} f(z)}{J_{z}^{\lambda} f(z)+(1-2 \alpha) J_{z}^{\lambda+1} f(z)}\right|<\beta \quad(z \in E) .
$$

The following observations are obvious:

(i) $\mathcal{S}(0, \alpha, 1)=\mathcal{S}^{*}(\alpha)$ is the class of starlike functions of order $\alpha$;

(ii) $\mathcal{S}(\lambda, \gamma, 1)=\mathcal{A}(\lambda+1, \lambda, \gamma)(0 \leq \lambda<1,-\lambda /(1-\lambda) \leq \gamma<1)$, the class studied by Kim and Srivastava [7].

In the present paper, we derive various properties and characteristics of the class $\mathcal{S}(a, c, A, B)$ by using the techniques of Briot-Bouquet differential 
subordination. We also obtain a sufficient condition, coefficient estimates and distortion theorems for this class. Further, we give some applications of our results to a class of fractional calculus operators. Many of our results improve and generalize the corresponding ones in [2], [3], and [7].

2. Preliminaries. In order to establish our results, we need the following lemmas.

Lemma 1 [8]. If $-1 \leq B<A \leq 1, \beta>0$ and the complex number $\gamma$ satisfy $\operatorname{Re}(\gamma) \geq-\beta(1-A) /(1-B)$, then the differential equation

$$
q(z)+\frac{z q^{\prime}(z)}{\beta q(z)+\gamma}=\frac{1+A z}{1+B z}
$$

has a univalent solution in $E$ given by

$$
q(z)= \begin{cases}\frac{z^{\beta+\gamma}(1+B z)^{\beta(A-B) / B}}{\beta \int_{0}^{z} t^{\beta+\gamma-1}(1+B t)^{\beta(A-B) / B} d t}-\frac{\gamma}{\beta}, & B \neq 0, \\ \frac{z^{\beta+\gamma} \exp (\beta A z)}{\beta \int_{0}^{z} t^{\beta+\gamma-1} \exp (\beta A t) d t}-\frac{\gamma}{\beta}, & B=0 .\end{cases}
$$

If $p(z)$ is analytic in $E$ and satisfies

$$
p(z)+\frac{z p^{\prime}(z)}{\beta p(z)+\gamma} \prec \frac{1+A z}{1+B z}
$$

then

$$
p(z) \prec q(z) \prec \frac{1+A z}{1+B z}
$$

and $q(z)$ is the best dominant.

Lemma $2[16]$. Let $\mu$ be a positive measure on the unit interval $[0,1]$. Let $g(t, z)$ be an analytic function in $E$ for each $t \in[0,1]$, and integrable in $t$ for each $z \in E$ and for almost all $t \in[0,1]$, and suppose that $\operatorname{Re}\{g(t, z)\}>0$ on $E, g(t,-r)$ is real and $\operatorname{Re}\{1 / g(t, z)\} \geq 1 / g(t,-r)$ for $|z| \leq r$ and $t \in[0,1]$. If $g(z)=\int_{0}^{z} g(t, z) d \mu(t)$, then $\operatorname{Re}\{1 / g(z)\} \geq 1 / g(-r)$ for $|z| \leq r$.

For real or complex numbers $\alpha_{1}, \alpha_{2}$ and $\beta_{1}\left(\beta_{1} \neq 0,-1,-2, \ldots\right)$, the hypergeometric function ${ }_{2} F_{1}(z)$ is defined by

$$
{ }_{2} F_{1}(z)={ }_{2} F_{1}\left(\alpha_{1}, \alpha_{2} ; \beta_{1} ; z\right)=\sum_{n=0}^{\infty} \frac{\left(\alpha_{1}\right)_{n}\left(\alpha_{2}\right)_{n}}{\left(\beta_{1}\right)_{n}} \frac{z^{n}}{n !} .
$$

We note that the series in (2.2) converges absolutely in $E$ (cf. [15]). The following identities are well known [15]. 
Lemma 3. For real or complex $\alpha_{1}, \alpha_{2}$ and $\beta_{1}\left(\beta_{1} \neq 0,-1,-2, \ldots\right)$, we have

$$
\begin{aligned}
& \text { (2.3) } \quad \int_{0}^{1} t^{\alpha_{2}-1}(1-t)^{\beta_{1}-\alpha_{2}-1}(1-t z)^{-\alpha_{1}} d t \\
& \quad=\frac{\Gamma\left(\alpha_{2}\right) \Gamma\left(\beta_{1}-\alpha_{2}\right)}{\Gamma\left(\beta_{1}\right)}{ }_{2} F_{1}\left(\alpha_{1}, \alpha_{2} ; \beta_{1} ; z\right) \quad\left(\operatorname{Re} \alpha_{1}>\operatorname{Re} \alpha_{2}>0\right), \\
& (2.4) \quad{ }_{2} F_{1}\left(\alpha_{1}, \alpha_{2} ; \beta_{1} ; z\right)={ }_{2} F_{1}\left(\alpha_{2}, \alpha_{1} ; \beta_{1} ; z\right), \\
& (2.5) \quad{ }_{2} F_{1}\left(\alpha_{1}, \alpha_{2} ; \beta_{1} ; z\right)=(1-z)^{-\alpha_{1}}{ }_{2} F_{1}\left(\alpha_{1}, \beta_{1}-\alpha_{2} ; \beta_{1} ; z\right) .
\end{aligned}
$$

LEMmA 4. Let $p(z)$ be analytic in $E$ with $p(0)=1$ and $p(z) \neq 0$ for $0<|z|<1$, and let $-1 \leq B<A \leq 1$.

(i) Let $B \neq 0$ and $\mu$ be a complex number with $\mu \neq 0$. Let $A, B$ and $\mu$ satisfy either

$$
\left|\mu \frac{A-B}{B}-1\right| \leq 1 \quad \text { or } \quad\left|\mu \frac{A-B}{B}+1\right| \leq 1 .
$$

If $p(z)$ satisfies

$$
1+\frac{z p^{\prime}(z)}{\mu p(z)} \prec \frac{1+A z}{1+B z} \quad(z \in E)
$$

then

$$
p(z) \prec q(z)=(1+B z)^{\mu(A-B) / B}
$$

and $q(z)$ is the best dominant.

(ii) Let $B=0, \mu$ be a complex number with $\mu \neq 0$, and $|\mu A|<\pi$. If $p(z)$ satisfies

$$
1+\frac{z p^{\prime}(z)}{\mu p(z)} \prec \frac{1+A z}{1+B z} \quad(z \in E)
$$

then

$$
p(z) \prec \exp (\mu A z)
$$

and this is the best dominant.

To avoid repetition we lay down, once for all, that $a>0, c>1,0 \leq \lambda<1$, $0 \leq \alpha<1,0<\beta \leq 1,-1 \leq B<A \leq 1$.

\section{Main results}

Theorem 1. If $B<A \leq(c-B) /(c-A)$, then:

(i) $\mathcal{S}(a, c, A, B) \subset \mathcal{S}\left(a, c+1, A^{*}, B\right)$ where $A^{*}=\{(c-1) A+B\} / c$. Furthermore, if $f \in \mathcal{S}(a, c, A, B)$ then

$$
\frac{\mathcal{L}(a, c) f(z)}{\mathcal{L}(a, c+1) f(z)} \prec \frac{1}{c Q(z)}=\widetilde{q}(z) \quad(z \in E)
$$


where

$$
Q(z)= \begin{cases}\int_{0}^{1} t^{c-1}\left(\frac{1+B t z}{1+B z}\right)^{(c-1)(A-B) / B} d t, & B \neq 0 \\ \int_{0}^{1} t^{c-1} \exp \{(c-1)(t-1) A z\} d t, & B=0,\end{cases}
$$

and $\widetilde{q}(z)$ is the best dominant.

(ii) If $B<0, A \leq \min \{(c-B) /(c-1),-2 B /(c-1)\}$ then for $f \in$ $\mathcal{S}(a, c, A, B)$,

$$
\begin{aligned}
\operatorname{Re} & \left\{\frac{\mathcal{L}(a, c) f(z)}{\mathcal{L}(a, c+1) f(z)}\right\} \\
& >\left\{{ }_{2} F_{1}\left(1, \frac{(c-1)(A-B)}{B} ; c+1 ; \frac{B}{B-1}\right)\right\}^{-1} \quad(z \in E) .
\end{aligned}
$$

The result is best possible.

Proof. From (1.5), it follows that

$$
z(\mathcal{L}(a, c) f(z))^{\prime}=(c-1) \mathcal{L}(a, c-1) f(z)+(2-c) \mathcal{L}(a, c) f(z) .
$$

Let $f \in \mathcal{S}(a, c, A, B)$. Setting

$$
p(z)=\frac{\mathcal{L}(a, c) f(z)}{\mathcal{L}(a, c+1) f(z)},
$$

we see that $p(z)$ is analytic in $E$ and $p(0)=1$. Making use of logarithmic differentiation in (3.4) and using the identity (3.3) in the resulting equation, we get

$$
P(z)+\frac{z P^{\prime}(z)}{(c-1) P(z)+1} \prec \frac{1+A z}{1+B z} \quad(z \in E)
$$

where $P(z)=\{c p(z)-1\} /(c-1)$. Using Lemma 1, we deduce that

$$
P(z) \prec q(z) \prec \frac{1+A z}{1+B z} \quad(z \in E)
$$

where $q(z)$ is the best dominant of (3.5) and is given by (2.1) for $\beta=c-1$ and $\gamma=1$. Again by (3.6), we obtain

$$
p(z) \prec \frac{1}{c Q(z)}=\widetilde{q}(z) \quad(z \in E),
$$

where $Q(z)$ is given by (3.1). This proves the first part of the theorem.

Now we prove (ii). We show that

$$
\inf _{|z|<1}\{\operatorname{Re}(\widetilde{q}(z))\}=\widetilde{q}(-1) .
$$


If we set $\alpha_{1}=\{(c-1)(B-A)\} / B, \alpha_{2}=c, \beta_{1}=c+1$, then $\beta_{1}>\alpha_{2}>0$. From (3.1), by using (2.3)-(2.5) we see that for $B \neq 0$,

$$
\begin{aligned}
Q(z) & =(1+B z)^{\alpha_{1}} \int_{0}^{1} t^{\alpha_{2}-1}(1+B t z)^{-\alpha_{1}} d t \\
& =\frac{\Gamma\left(\alpha_{2}\right) \Gamma\left(\beta_{1}-\alpha_{2}\right)}{\Gamma\left(\beta_{1}\right)}{ }_{2} F_{1}\left(1, \alpha_{1} ; \beta_{1} ; \frac{B z}{1+B z}\right) .
\end{aligned}
$$

To prove (3.7), we show that $\operatorname{Re}\{1 / Q(z)\} \geq 1 / Q(-1), z \in E$. Again, by (3.8) for $B<0, A<-2 B /(c-1)$ (so that $\beta_{1}>\alpha_{1}>0$ ), (3.1) can be written as

$$
Q(z)=\int_{0}^{1} g(t, z) d \mu(t)
$$

where

$$
g(t, z)=\frac{1+B z}{1+(1-t) B z}
$$

and

$$
d \mu(t)=\frac{\Gamma\left(\alpha_{2}\right)}{\Gamma\left(\alpha_{1}\right) \Gamma\left(\beta_{1}-\alpha_{1}\right)} t^{\alpha_{1}-1}(1-t)^{\beta_{1}-\alpha_{1}-1} d t
$$

is a positive measure on $[0,1]$.

For $-1 \leq B<0$, it may be noted that $\operatorname{Re}\{g(t, z)\}>0, g(t,-r)$ is real for $0 \leq r<1, t \in[0,1]$ and

$$
\operatorname{Re}\left\{\frac{1}{g(t, z)}\right\} \geq \frac{1-(1-t) B r}{1-B r}=\frac{1}{g(t,-r)} \quad(|z| \leq r<1, t \in[0,1]) .
$$

Therefore, by using Lemma 2, we deduce that $\operatorname{Re}\{1 / Q(z)\} \geq 1 / Q(-r)$, $|z| \leq r<1$ and by taking $r \rightarrow 1^{-}$, we obtain $\operatorname{Re}\{1 / Q(z)\} \geq 1 / Q(-1)$, $z \in E$. In the case $A=-2 B /(c-1)$, we obtain the required assertion by letting $A \rightarrow(-2 B /(c-1))^{+}$. This proves (3.7).

The result is best possible because of the best dominant property of $\widetilde{q}(z)$.

Putting $a=2, c=2-\lambda, A=\beta(1-2 \alpha)$ and $B=-\beta$ in Theorem 1, we get

Corollary 1. If $f \in \mathcal{S}(\lambda, \alpha, \beta)$, then

$$
\operatorname{Re}\left\{\frac{J_{z}^{\lambda} f(z)}{J_{z}^{\lambda-1} f(z)}\right\}>\left\{{ }_{2} F_{1}\left(1,2(1-\lambda)(1-\alpha) ; 3-\lambda ; \frac{\beta}{\beta+1}\right)\right\}^{-1} \quad(z \in E) .
$$

The result is best possible.

For $\lambda=0$ and $\beta=1$, Corollary 1 yields 
Corollary 2. If $f \in \mathcal{S}^{*}(\alpha)$, then

$$
\operatorname{Re}\left\{z f(z)\left(\int_{0}^{z} f(t) d t\right)^{-1}\right\}>2\left({ }_{2} F_{1}(1,2(1-\alpha) ; 3 ; 1 / 2)\right)^{-1} \quad(z \in E) .
$$

The result is best possible.

Theorem 2. Let $f \in \mathcal{S}(a, c, A, B)$, where $-1 \leq B<A \leq 1(B \neq 0)$. If either

$$
\left|(c-1) \frac{A-B}{B}-1\right| \leq 1 \quad \text { or } \quad\left|(c-1) \frac{A-B}{B}+1\right| \leq 1
$$

then

$$
\frac{\mathcal{L}(a, c) f(z)}{z} \prec(1+B z)^{(c-1)(A-B) / B} \quad(z \in E) .
$$

In case $B=0$, i.e., for $f \in \mathcal{S}(a, c, A, 0)(0<A \leq 1)$, we have

$$
\frac{\mathcal{L}(a, c) f(z)}{z} \prec \exp ((c-1) A z) \quad(z \in E),
$$

where $|A|<\pi /(c-1)$. The result is best possible.

Proof. Setting $p(z)=(\mathcal{L}(a, c) f(z)) / z$, we note that $p(z)$ is analytic in $E$, $p(0)=1$ and $p(z) \neq 0$ for $z \in E$. Logarithmic differentiation $p(z)$ followed by the use of the identity (3.3) yields

$$
1+\frac{z p^{\prime}(z)}{(c-1) p(z)}=\frac{\mathcal{L}(a, c-1) f(z)}{\mathcal{L}(a, c) f(z)} \prec \frac{1+A z}{1+B z} \quad(z \in E) .
$$

For such $p(z)$, from (i) and (ii) of Lemma 4, we get the relations (3.9) and (3.10) of the theorem.

COROLlaRY 3. Under the hypotheses of Theorem 2, we have, for $|z|=$ $r<1$,

$$
|\mathcal{L}(a, c) f(z)| \leq \begin{cases}r(1+B r)^{(c-1)(A-B) / B}, & B \neq 0, \\ r \exp ((c-1) A r), & B=0,\end{cases}
$$

and

$$
|\mathcal{L}(a, c) f(z)| \geq \begin{cases}r(1-B r)^{(c-1)(A-B) / B}, & B \neq 0, \\ r \exp (-(c-1) A r), & B=0 .\end{cases}
$$

All the above estimates are sharp.

Proof. For $B \neq 0$, we deduce from (3.9) that

$$
\frac{\mathcal{L}(a, c) f(z)}{z}=(1+B \omega(z))^{(c-1)(A-B) / B},
$$

where $\omega(z)$ is analytic in $E$ satisfying the conditions $\omega(0)=0$ and $|\omega(z)|$ $\leq|z|$ for $z \in E$. 
(i) When $B>0$,

$$
\begin{aligned}
\left|\frac{\mathcal{L}(a, c) f(z)}{z}\right| & =\left|(1+B \omega(z))^{(c-1)(A-B) / B}\right| \\
& =\left|\exp \left[\frac{(c-1)(A-B)}{B} \log (1+B \omega(z))\right]\right| \\
& =\exp \left[\operatorname{Re}\left\{\frac{(c-1)(A-B)}{B} \log (1+B \omega(z))\right\}\right] \\
& =\exp \left[\frac{(c-1)(A-B)}{B} \log |(1+B \omega(z))|\right] \\
& \leq \mid\left(1+\left.B \omega(z)\right|^{(c-1)(A-B) / B} \leq(1+B r)^{(c-1)(A-B) / B} .\right.
\end{aligned}
$$

(ii) When $B<0$, we put $B=-D, D>0$, so that

$$
\begin{aligned}
\left|\frac{\mathcal{L}(a, c) f(z)}{z}\right| & =\left|(1+B \omega(z))^{(c-1)(A-B) / B}\right|=\left|\left\{(1-D \omega(z))^{-1}\right\}^{(c-1)(A-B) / B}\right| \\
& \leq\left|(1-D \omega(z))^{-1}\right|^{(c-1)(A-B) / B} \leq\left(\frac{1}{1-D r}\right)^{(c-1)(A-B) / B} \\
& \leq(1+B r)^{(c-1)(A-B) / B} .
\end{aligned}
$$

In case $B=0$ and $|A|<\pi /(c-1)$, we have

$$
\left|\frac{\mathcal{L}(a, c) f(z)}{z}\right|=\exp \{(c-1) A \operatorname{Re}(\omega(z))\} \leq \exp \{(c-1) A r\} .
$$

This proves the assertion (3.11). Similarly, we can prove (3.12).

The bounds are sharp, being attained by the function $f(z)$ defined by

$$
\mathcal{L}(a, c) f(z)= \begin{cases}z\left(1+B \delta_{1} z\right)^{(c-1)(A-B) / B}, & B \neq 0, \\ z \exp ((c-1) A z), & B=0 .\end{cases}
$$

For $a=2, c=2-\lambda, A=\beta(1-2 \alpha)$ and $B=-\beta$, Corollary 3 yields

Corollary 4. If $f \in \mathcal{S}(\lambda, \alpha, \beta)$, then for $|z|=r<1$,

$$
\frac{r}{(1+\beta r)^{2(1-\lambda)(1-\alpha)}} \leq\left|J_{z}^{\lambda}(z)\right| \leq \frac{r}{(1-\beta r)^{2(1-\lambda)(1-\alpha)}} .
$$

The bounds are sharp.

Corollary 5. If $f \in \mathcal{S}(\lambda, \alpha, \beta)$, then

$$
\operatorname{Re}\left\{\frac{J_{z}^{\lambda}(z)}{z}\right\}>(1+\beta)^{-2(1-\lambda)(1-\alpha)} \quad(z \in E) .
$$

The result is sharp.

TheOREM 3. Let $\delta$ be a real number satisfying

$$
B<A \leq B+\frac{(1-B)(\delta+1)}{c-1} .
$$


(i) If $f \in \mathcal{S}(a, c, A, B)$, then the function $\mathcal{F}_{\delta}$ defined by (1.2) belongs to the class $\mathcal{S}(a, c, A, B)$. Furthermore,

$$
\frac{\mathcal{L}(a, c-1) \mathcal{F}_{\delta}(z)}{\mathcal{L}(a, c) \mathcal{F}_{\delta}(z)} \prec \frac{1}{c-1}\left[\frac{1}{Q(z)}-(2+\delta-c)\right] \equiv \widetilde{q}(z) \quad(z \in E)
$$

where

$$
Q(z)= \begin{cases}\int_{0}^{1} t^{\delta}\left(\frac{1+B t z}{1+B z}\right)^{(c-1)(A-B) / B} d t, & B \neq 0, \\ \int_{0}^{1} t^{\delta} \exp \{(c-1)(t-1) A z\} d t, & B=0,\end{cases}
$$

and $\widetilde{q}(z)$ is the best dominant.

(ii) If $B<0$ and

$$
A \leq \min \left\{\frac{(1-B)(\delta+1)}{c-1}+B,-\frac{(\delta+3-c) B}{c-1}\right\}
$$

then for $f \in \mathcal{S}(a, c, A, B)$, we have

$$
\begin{aligned}
\operatorname{Re}\left\{\frac{\mathcal{L}(a, c-1) \mathcal{F}_{\delta}(z)}{\mathcal{L}(a, c) \mathcal{F}_{\delta}(z)}\right\} & \\
> & \frac{1}{c-1}\left[(\delta+1)\left\{{ }_{2} F_{1}\left(1, \frac{(c-1)(A-B)}{B} ; \delta+2 ; \frac{B}{B-1}\right)\right\}^{-1}\right. \\
& \quad-(2+\delta-c)] \quad(z \in E) .
\end{aligned}
$$

The result is best possible.

Proof. Since $\mathcal{F}_{\delta}(z)=z+\sum_{n=2}^{\infty}\{(\delta+1) /(\delta+n)\} a_{n} z^{n}$, it follows from (1.5) that

$$
z\left(\mathcal{L}(a, c) \mathcal{F}_{\delta}(z)\right)^{\prime}=(\delta+1) \mathcal{L}(a, c) f(z)-\delta \mathcal{L}(a, c) \mathcal{F}_{\delta}(z)
$$

Putting

$$
p(z)=\frac{\mathcal{L}(a, c-1) \mathcal{F}_{\delta}(z)}{\mathcal{L}(a, c) \mathcal{F}_{\delta}(z)},
$$

we see that $p(z)$ is analytic in $E$ with $p(0)=1$. Since $f \in \mathcal{S}(a, c, A, B)$, it is clear that $\mathcal{L}(a, c) f(z) \neq 0$ in $0<|z|<1$ so that (3.3) and (3.16) give

$$
\frac{\mathcal{L}(a, c) \mathcal{F}_{\delta}(z)}{\mathcal{L}(a, c) f(z)}=\frac{\delta+1}{(c-1) p(z)+(2+\delta-c)} \quad(z \in E) .
$$

Making use of the logarithmic differentiation in (3.18) and using (3.17), we deduce that

$$
p(z)+\frac{z p^{\prime}(z)}{(c-1) p(z)+(2+\delta-c)}=\frac{\mathcal{L}(a, c-1) f(z)}{\mathcal{L}(a, c) f(z)} \prec \frac{1+A z}{1+B z} \quad(z \in E) .
$$


Using Lemma 1, we obtain

$$
p(z) \prec \frac{1}{c-1}\left[\frac{1}{Q(z)}-(2+\delta-c)\right] \equiv \widetilde{q}(z) \prec \frac{1+A z}{1+B z} \quad(z \in E),
$$

where $Q(z)$ is given by $(3.15)$, and $\widetilde{q}(z)$ is the best dominant. This proves the first part of the theorem.

Proceeding as in Theorem 1 we get the second part.

Taking $a=2, c=2-\lambda, A=\beta(1-2 \alpha)$ and $B=-\beta$ in Theorem 3 , we obtain

Corollary 6. Let $\delta$ be a real number satisfying $\delta \geq\{(1-\lambda)(1-2 \alpha)-$ $(1+\beta \lambda)\} /(1+\beta)$.

(i) If $f \in \mathcal{S}(\lambda, \alpha, \beta)$, then the function $\mathcal{F}_{\delta}$ defined by (1.2) belongs to the class $\mathcal{S}(\lambda, \alpha, \beta)$. Furthermore,

$$
\frac{J_{z}^{1+\lambda} \mathcal{F}_{\delta}(z)}{J_{z}^{\lambda} \mathcal{F}_{\delta}(z)} \prec \frac{1}{1-\lambda}\left\{\frac{1}{Q(z)}-(\delta+\lambda)\right\} \equiv \widetilde{q}(z) \quad(z \in E)
$$

where $Q(z)$ is obtained from (3.15) for $c=2-\lambda, A=\beta(1-2 \alpha)$ and $B=-\beta$.

(ii) If

$$
\delta \geq \max \left\{\frac{(1-\lambda)(1-2 \alpha)-(1+\beta \lambda)}{1+\beta}, \frac{(1-\lambda)(1-2 \alpha)-\beta(1+\lambda)}{\beta}\right\}
$$

and $f \in \mathcal{S}(\lambda, \alpha, \beta)$, then $\mathcal{F}_{\delta} \in \mathcal{S}(\lambda, \varrho, \beta)$, where

$$
\varrho=\frac{1}{1-\lambda}\left[(\delta+1)\left\{{ }_{2} F_{1}\left(1,2(1-\lambda)(1-\alpha) ; \delta+2 ; \frac{\beta}{\beta+1}\right)\right\}^{-1}-(\delta+\lambda)\right] .
$$

The result is best possible.

Remark. Substituting $\lambda=0$ and $\beta=1$ in part (ii) of Corollary 6 , we see that $f \in \mathcal{S}^{*}(\alpha)(0 \leq \alpha<1)$ implies that $\mathcal{F}_{\delta} \in \mathcal{S}^{*}\left(\varrho_{2}\right)$, where $\varrho_{2}=(\delta+1)\left\{{ }_{2} F_{1}(1,2(1-\alpha) ; \delta+2 ; 1 / 2)\right\}^{-1}-\delta$, provided $\delta \geq-\alpha$. This is an improvement of a recent result of Bajpai and Srivastava [2] and Bernardi [3] for $\delta=1,2, \ldots$.

TheOrem 4. Let $f \in \mathcal{A}$ be given by (1.1) and $-1 \leq B<0$. If

$$
\sum_{n=2}^{\infty} \frac{\{(1-B)(n-1)+(A-B)(c-1)\}(a)_{n}}{(c-1)_{n}}\left|a_{n}\right| \leq A-B
$$

then $f \in \mathcal{S}(a, c, A, B)$. The result is sharp.

Proof. Suppose (3.19) holds. Then for $|z|=r<1$, 


$$
\begin{aligned}
\mid \mathcal{L}(a, c-1) & f(z)-\mathcal{L}(a, c) f(z)|-| A \mathcal{L}(a, c) f(z)-B \mathcal{L}(a, c-1) f(z) \mid \\
\leq & \sum_{n=2}^{\infty} \frac{(n-1)(a)_{n}}{(c-1)_{n}}\left|a_{n}\right| r^{n} \\
& -\left\{(A-B) r-\sum_{n=2}^{\infty} \frac{\{(A-B)(c-1)-(n-1) B\}(a)_{n}}{(c-1)_{n}}\left|a_{n}\right| r^{n}\right\} \\
< & \sum_{n=2}^{\infty} \frac{(n-1)(a)_{n}}{(c-1)_{n}}\left|a_{n}\right| \\
& -\left\{(A-B)-\sum_{n=2}^{\infty} \frac{\{(A-B)(c-1)-(n-1) B\}(a)_{n}}{(c-1)_{n}}\left|a_{n}\right|\right\} \\
= & \sum_{n=2}^{\infty} \frac{\{(1-B)(n-1)+(A-B)(c-1)\}(a)_{n}}{(c-1)_{n}}\left|a_{n}\right|-(A-B) \leq 0 .
\end{aligned}
$$

Thus, it follows from (1.7) that $f \in \mathcal{S}(a, c, A, B)$.

The result is sharp for the functions

$$
f_{n}(z)=z+\sum_{n=2}^{\infty} \frac{(A-B)(c-1)_{n}}{\{(1-B)(n-1)+(A-B)(c-1)\}(a)_{n}} z^{n} \quad(n \geq 2),
$$

because

$$
\left|\frac{\mathcal{L}(a, c-1) f_{n}(z)-\mathcal{L}(a, c) f_{n}(z)}{A \mathcal{L}(a, c) f_{n}(z)-B \mathcal{L}(a, c-1) f_{n}(z)}\right|=1 \quad \text { for } z=\exp (i \pi / n) .
$$

Corollary 7. Let $f \in \mathcal{A}$ be given by (1.1). If

$$
\sum_{n=2}^{\infty} \frac{\Gamma(n+1) \Gamma(1-\lambda)\{(1+\beta)(n-1)+2 \beta(1-\alpha)\}}{\Gamma(n+1-\lambda)}\left|a_{n}\right| \leq 2 \beta(1-\alpha)
$$

then $f \in \mathcal{S}(\lambda, \alpha, \beta)$. The result is sharp.

TheOREM 5. If $f$ given by (1.1) belongs to $\mathcal{S}(a, c, A, B)$, then

$$
\left|a_{n}\right| \leq \frac{(A-B)(c-1)_{n}}{(n-1)(a)_{n-1}} \prod_{j=2}^{n-1}\left(1+\frac{(A-B)(c-1)}{j-1}\right) \quad(n \geq 2) .
$$

The result is sharp.

Proof. Since $f \in \mathcal{S}(a, c, A, B)$, we have

$$
\mathcal{L}(a, c-1) f(z)=p(z) \mathcal{L}(a, c) f(z)
$$

where $p(z)=1+p_{1} z+p_{2} z^{2}+\ldots \in \mathcal{P}(A, B)$. Substituting the power series expansions of $\mathcal{L}(a, c-1) f(z), \mathcal{L}(a, c) f(z)$ and $p(z)$ in (3.21) and comparing the coefficients of $z^{n}$ on both sides of the resulting equation, we obtain 


$$
\frac{(n-1)(a)_{n-1}}{(c-1)_{n}} a_{n}=p_{n-1}+\frac{(a)_{1}}{(c)_{1}} p_{n-2} a_{2}+\ldots+\frac{(a)_{n-2}}{(c)_{n-2}} p_{1} a_{n-1} .
$$

Using the fact [1] that

$$
\left|p_{n}\right| \leq A-B \quad(n \geq 1)
$$

in (3.22), we get

$$
\frac{(n-1)(a)_{n-1}}{(c-1)_{n}}\left|a_{n}\right| \leq(A-B)\left\{1+\sum_{m=2}^{n-1} \frac{(a)_{m-1}}{(c)_{m-1}}\left|a_{m}\right|\right\} .
$$

We will prove by induction that the assertion (3.20) is satisfied for $n \geq 2$. If $n=2$, then

$$
\left|a_{2}\right| \leq \frac{(c-1)_{2}(A-B)}{(a)_{1}} .
$$

Now suppose that (3.20) is satisfied for $n \leq k$. Then, from (3.23), we have

$$
\begin{aligned}
& \frac{k(a)_{k}}{(c-1)_{k+1}}\left|a_{k+1}\right| \leq(A-B)\left\{1+\sum_{m=2}^{k} \frac{(a)_{m-1}}{(c)_{m-1}}\left|a_{m}\right|\right\} \\
& \leq(A-B)\left\{1+\sum_{m=2}^{k} \frac{(A-B)(c-1)}{m-1} \prod_{j=2}^{m-1}\left(1+\frac{(A-B)(c-1)}{j-1}\right)\right\} \\
& =(A-B) \prod_{j=2}^{k}\left(1+\frac{(A-B)(c-1)}{j-1}\right) .
\end{aligned}
$$

Hence

$$
\left|a_{n}\right| \leq \frac{(A-B)(c-1)_{n}}{(n-1)(a)_{n-1}} \prod_{j=2}^{n-1}\left(1+\frac{(A-B)(c-1)}{j-1}\right)
$$

for all $n \geq 2$.

Finally, we note that the result is sharp for the functions $f_{n}(z)$ given by

$$
f_{n}(z)=z+\frac{(A-B)(c-1)_{n}}{(n-1)(a)_{n-1}} \prod_{j=2}^{n-1}\left(1+\frac{(A-B)(c-1)}{j-1}\right) z^{n} \quad(n \geq 2) .
$$

Corollary 8. If $f$, given by (1.1), belongs to the class $\mathcal{S}(\lambda, \alpha, \beta)$, then

$$
\left|a_{n}\right| \leq \frac{2 \beta(1-\alpha) \Gamma(n+1-\lambda)}{(n-1) \Gamma(n+1) \Gamma(1-\lambda)} \prod_{j=2}^{n-1}\left(1+\frac{2 \beta(1-\alpha)(1-\lambda)}{j-1}\right) \quad(n \geq 2) .
$$

The result is sharp. 
TheOREM 6. Let $f$, given by (1.1), belong to the class $\mathcal{S}(a, c, A, B)$ and $\mu$ be any complex number. Then

$$
\begin{aligned}
\left|a_{3}-\mu a_{2}^{2}\right| & \leq \frac{(A-B)(c-1)_{3}}{2(a)_{2}} \\
& \times \max \left\{1,\left|\{B-(A-B)(c-1)\}+\mu \frac{2(A-B)(a+1)(c-1)_{2}}{a(c+1)}\right|\right\} .
\end{aligned}
$$

The result is sharp.

Proof. For $f \in \mathcal{S}(a, c, A, B)$, we have, by (1.6),

$$
\begin{aligned}
& \text { (3.24) } \quad \sum_{n=2}^{\infty} \frac{(n-1)(a)_{n-1}}{(c-1)_{n}} a_{n} z^{n} \\
& =\left\{(A-B) z+\sum_{n=2}^{\infty} \frac{\{(A-B)(c-1)-(n-1) B\}(a)_{n-1}}{(c-1)_{n}} a_{n} z^{n}\right\}\left\{\sum_{j=1}^{\infty} \omega_{j} z^{j}\right\}
\end{aligned}
$$

where $\omega(z)=\sum_{j=1}^{\infty} \omega_{j} z^{j}$ is analytic in $E$ with $|\omega(z)|<1$ for $z \in E$. On equating the coefficients of $z^{2}$ and $z^{3}$ on both sides of (3.24), we deduce that

$$
a_{2}=\frac{(A-B)(c-1)}{a} \omega_{1}
$$

and

$$
a_{3}=\frac{(A-B)(c-1)}{2(a)_{2}}\left\{\omega_{2}+((A-B)(c-1)-B) \omega_{1}^{2}\right\} .
$$

It is known [6] that for every complex number $\gamma$,

$$
\left|\omega_{2}-\gamma \omega_{1}^{2}\right| \leq \max \{1,|\gamma|\}
$$

and the estimate is sharp. Now, by using (3.25) and (3.26), we obtain

$$
\left|a_{3}-\mu a_{2}^{2}\right| \leq \frac{(A-B)(c-1)_{3}}{2(a)_{2}}\left|\omega_{2}-\gamma \omega_{1}^{2}\right|,
$$

where

$$
\gamma=\{B-(A-B)(c-1)\}+\mu \frac{2(A-B)(a+1)(c-1)_{2}}{a(c+1)} .
$$

The assertion of the theorem follows by using (3.27) in (3.28). The result is sharp as the estimate (3.27) is sharp.

Corollary 9. If $f$, given by (1.1), belongs to the class $\mathcal{S}(\lambda, \alpha, \beta)$, then for any complex number $\mu$

$$
\begin{aligned}
\left|a_{3}-\mu a_{2}^{2}\right| & \leq \frac{\beta(1-\alpha) \Gamma(4-\lambda)}{3 ! \Gamma(1-\lambda)} \\
& \times \max \left\{1,\left|\frac{6 \beta(1-\alpha)(1-\lambda)(2-\lambda)}{3-\lambda}-\beta\{2(1-\alpha)(1-\lambda)+1\}\right|\right\} .
\end{aligned}
$$

The result is sharp. 


\section{References}

[1] V. Anh, K-fold symmetric starlike univalent functions, Bull. Austral. Math. Soc. 32 (1985), 419-436.

[2] S. K. Bajpai and R. S. L. Srivastava, On the radius of convexity and starlikeness of univalent functions, Proc. Amer. Math. Soc. 32 (1972), 153-160.

[3] S. D. Bernardi, Convex and starlike univalent functions, Trans. Amer. Math. Soc. 135 (1969), 429-446.

[4] B. C. Carlson and S. B. Shaffer, Starlike and prestarlike hypergeometric functions, SIAM J. Math. Anal. 15 (1984), 737-745.

[5] Z. Janowski, Some extremal problems for certain families of analytic functions, Ann. Polon. Math. 28 (1973), 297-326.

[6] F. R. Keogh and E. P. Merkes, A coefficient inequality for certain classes of analytic functions, Proc. Amer. Math. Soc. 20 (1960), 8-12.

[7] Y. C. Kim and H. M. Srivastava, Fractional integral and other linear operators associated with the Gaussian hypergeometric functions, Complex Variables Theory Appl. 34 (1997), 293-312.

[8] S. S. Miller and P. T. Mocanu, Univalent solutions of Briot-Bouquet differential equations, J. Differential Equations 58 (1985), 297-309.

[9] M. Obradović and S. Owa, On certain properties for some classes of starlike functions, J. Math. Anal. Appl. 145 (1990), 357-364.

[10] S. Owa, On the distortion theorems. I, Kyungpook Math. J. 18 (1978), 53-59.

[11] S. Ruscheweyh, New criteria for univalent functions, Proc. Amer. Math. Soc. 49 (1975), 109-115.

[12] S. G. Samko, A. A. Kilbas and O. I. Marichev, Fractional Integrals and Derivatives. Theory and Applications, Gordon and Breach, New York, 1993.

[13] H. M. Srivastava and R. G. Buschman, Theory and Applications of Convolution Integral Equations, Kluwer, Dordrecht, 1992.

[14] H. M. Srivastava and S. Owa (eds.), Univalent Functions, Fractional Calculus, and Their Applications, Ellis Horwood, Chichester, 1989.

[15] E. T. Whittaker and G. N. Watson, A Course of Modern Analysis, 4th edition (reprinted), Cambridge Univ. Press, Cambridge, 1962.

[16] D. R. Wilken and J. Feng, A remark on convex and starlike functions, J. London Math. Soc. 21 (1980), 287-290.

J. Patel

Department of Mathematics

Utkal University

Vani Vihar

Bhubaneswar 751004, India

E-mail: jpatelmath@sify.com
G. P. Mohapatra

Silicon School of Information Technology

Commercial Complex

Acharya Vihar

Bhubaneswar 751013, India 